\title{
Complicaciones intraoperatorias de la histerectomía vaginal por causas benignas. Experiencia de seis años, Hospital Clínico de la Universidad de Chile
}

\author{
Valentín Manríquez G. ${ }^{1}$, Michel Naser $N^{1}{ }^{1}$, Mauricio Gómez L. ${ }^{2}$ \\ ${ }^{1}$ Unidad de Piso Pélvico Femenino, Hospital Clínico Universidad de Chile. ${ }^{2}$ Unidad de Uroginecología y Piso Pélvico, \\ Clínica Universitaria Bolivariana, Universidad Pontificia Bolivariana, Medellín, Colombia.
}

\section{RESUMEN}

Antecedentes: La histerectomía es la cirugía en la mujer más frecuentemente realizada después de la cesárea a nivel mundial. La mayoría de las veces este procedimiento puede efectuarse por vía vaginal. Objetivo: Realizar un análisis retrospectivo de las histerectomías vaginales realizadas en la Unidad de Piso Pélvico del Hospital Clínico de la Universidad de Chile, sus principales indicaciones y las complicaciones intraoperatorias (2006-2012). Resultados: Se analizaron 379 histerectomías por causa benigna, el 17\% ( $n=64)$ por vía abdominal y el $83 \%(n=315)$ por vía vaginal. Las principales complicaciones intraoperatorias en las histerectomías vaginales, fueron lesiones vasculares $(1,2 \%)$ y vesicales $(0,6 \%)$. Hubo complicaciones infecciosas en el $1,2 \%$ de las pacientes (4 abscesos de la cúpula vaginal), las que han disminuido con el uso de los antibióticos profilácticos. Conclusiones: La histerectomía vaginal, es la vía ideal de abordaje quirúrgico para la patología benigna del útero, por menores complicaciones, rápida recuperación, costo efectividad y reintegro de las pacientes a sus actividades. Nuestros resultados de complicaciones son similares a los reportados por la literatura nacional e internacional.

\section{PALABRAS CLAVES: Histerectomía vaginal, complicaciones intraoperatorias}

\section{SUMMARY}

Background: Hysterectomy is the surgery most frequently in women after caesarean section performed worldwide. Most often, this procedure can be carried out vaginally. Objective: To perform a retrospective analysis of vaginal hysterectomies performed in Pelvic Floor Unit, Clinical Hospital of the University of Chile, the main indications and intraoperative complications (2006-2012). Results: We analyzed 379 hysterectomies for benign causes, $17 \%(n=64)$ for abdominal and $83 \%(n=315)$ vaginally. The major intraoperative complications in vaginal hysterectomies were vascular (1.2\%) and bladder lesions ( $0.6 \%)$. Infectious complications occurred in $1.2 \%$ of patients (4 vaginal vault abscess), which decreased with the use of prophylactic antibiotics. Conclusions: Vaginal hysterectomy is the ideal way of surgical approach for benign disease of the uterus, fewer complications, faster recovery, cost-effectiveness and reimbursement of patients to their activities. Our results and complications are similar to those reported by national and international literature.

KEY WORDS: Vaginal hysterectomy, intraoperative complications 


\section{INTRODUCCIÓN}

La histerectomía, es la cirugía que con mayor frecuencia se realiza luego de la cesárea en los Estados Unidos de Norte América $(1,2)$. Allí se realizan más de 600.000 histerectomías cada año (3), contabilizándose entre el año 2000 y 2004 3,1 millones de histerectomías (4).

Usualmente la relación histerectomía vaginal vs histerectomía abdominal es de 1:3 o 1:4 según el país, el sitio en el que se realiza y la experiencia de los cirujanos en procedimientos vaginales (2). Recientes reportes demuestran que la histerectomía abdominal se realiza en $66,1 \%$ de los casos, seguida por la vía vaginal y por último la laparoscópica con el $11,8 \%$ de los casos (5).

La histerectomía vaginal es segura, amigable para la paciente y económicamente conveniente. Está indicada en la mayoría de las pacientes con enfermedad benigna, principalmente en pacientes que tienen cicatrices abdominales por herniorrafias, obesidad mórbida y patologías cardiopulmonares, entre otras (6).

Diversos estudios, han demostrado las ventajas de la histerectomía vaginal, sobre la histerectomía abdominal, dentro de las que se destacan ser más segura, económica, de menor morbilidad y mejor tolerancia por parte de las pacientes con alto riesgo quirúrgico o de mayor edad $(6,7)$. Es así como en 2008 el Colegio Americano de Obstetras y Ginecólogos concluyó en un reporte bibliográfico del tema, que la histerectomía vaginal está asociada con mejores resultados y menores complicaciones que la histerectomía abdominal o laparoscópica (4). También, la revisión de la base Cochrane de 2010 analiza los resultados de 34 estudios aleatorios controlados de histerectomía vaginal, laparoscópica y abdominal, concluyendo que los mejores resultados desde el punto de vista clínico son los obtenidos por la cirugía vaginal. Cuando no es posible realizar la histerectomía vaginal, la histerectomía laparoscópica tiene ventajas comparativas respecto a la histerectomía abdominal (8).

Las principales indicaciones de histerectomía son la hemorragia uterina disfuncional, la adenomiosis, los miomas uterinos, la endometriosis y el prolapso de los órganos pélvicos $(3,9)$. Dentro de las principales indicaciones sugeridas para la realización de una histerectomía vaginal cuentan las cicatrices de herniorrafias, la obesidad mórbida, la cicatrización queloide y la sospecha de alto riesgo de complicaciones en la cirugía por vía abdominal (10).

Aunque no existen contraindicaciones absolutas para la realización de la histerectomía vaginal, existen condiciones que afectan la decisión del cirujano en el momento de decidir la vía de abordaje, entre ellas se mencionan como principales: entrenamiento del cirujano y experiencia, otras enfermedades extrauterinas (patología anexial, endometriosis severa, síndrome adherencial), tamaño y forma del útero, necesidad de otros procedimientos asociados, y preferencia de la paciente.

Algunos autores refieren las siguientes características como posibles contraindicaciones para la histerectomía vaginal (11): útero de tamaño mayor a un embarazo de 12 semanas o mayor de $300 \mathrm{cc}$ (mayor importancia el volumen que el tamaño), nuliparidad, cesárea previa, vagina estrecha, ángulo subpúbico menor de 90 grados, útero fijo.

La necesidad de realizar anexectomía no debe ser una contraindicación de la histerectomía vaginal. Esta técnica, sólo tiene entre un 3 y $4 \%$ de fracaso por vía vaginal y su éxito depende tanto de la experiencia del cirujano así como de la necesidad de contar con el material quirúrgico adecuado $(12,13,14)$.

Ante la duda realizar la cirugía por vía vaginal, las pacientes deberían ser sometidas a un examen pélvico bajo anestesia para una mejor valoración, descartar otras patologías pélvicas y asegurar la factibilidad de ésta (15). En algunos casos que existen dudas acerca de la vía para la histerectomía, puede ser útil la realización de una laparoscopia diagnóstica, la cual ayuda a valorar los hallazgos pélvicos y a tomar una decisión con base en estos (15).

Como ayuda para minimizar las posibles complicaciones de la vía urinaria baja, la tendencia es a generalizar de rutina la cistoscopia, luego de cirugía ginecológica mayor $(15,16)$. Con la adecuada elección de las pacientes, el adecuado entrenamiento, la correcta valoración clínica y pre quirúrgica, la vía vaginal, se recomienda en el $80 \%$ de las histerectomías (17).

Se mencionan las siguientes consideracionesque pueden dificultar o hacer abandonar la vía vaginal: mioma cervical, mucosa vaginal que envuelve el labio anterior del cérvix, cirugía previa (incluye cesárea), endometriosis severa, tuberculosis peritoneal, mucosa vaginal que envuelve el labio posterior del cérvix.

Las complicaciones intraoperatorias primarias son daños viscerales y vasculares. Las tasas reportadas de hemorragia varían entre el 1,4 y el $2,6 \%$ y las de daño ureteral y vesical entre el 0,88 y el $1,76 \%$ respectivamente (18).

La complicación postquirúrgica más importante, es la infección pélvica. La enfermedad febril post quirúrgica ocurre en aproximadamente el $15 \%$ de las pacientes y puede ser disminuida con el uso de antibióticos profilácticos. Las infecciones post operatorias de una histerectomía vaginal incluyen la celulitis de cúpula, celulitis pélvica, el absceso pélvico; estas infecciones ocurren en aproximadamente el $4 \%$ de las pacientes (19). 
La experiencia en nuestra unidad incluye la realización de histerectomía vaginal por causa benigna, sobre la histerectomía abdominal. Independiente si existe prolapso genital o no. La elección de la vía abdominal, está determinada por úteros de tamaño mayor a $15 \mathrm{~cm}$, la presencia de un útero adherido no móvil, el antecedente de cirugías pélvicas importantes, y que además al examen físico se encuentre un útero poco móvil y doloroso, condiciones que hacen pensar en síndrome adherencial. Otra causa para la necesidad de realizar la histerectomía por vía abdominal, es la necesidad de realizar colposacropexia debido al diagnóstico de prolapso apical en pacientes menores de 65 años, activas física y sexualmente.

El objetivo del estudio es realizar una revisión retrospectiva de todas las histerectomías vaginales realizadas en la Unidad de Piso Pélvico Femenino del Hospital Clínico de la Universidad de Chile durante los últimos 6 años, para describir sus principles indicaciones y complicaciones intraoperatorias.

\section{PACIENTES Y MÉTODOS}

Se realizó una revisión de todas las historias clínicas electrónicas que posee la Unidad de Piso Pélvico Femenino del Hospital Clínico de la Universidad de Chile. Se seleccionaron las pacientes sometidas a histerectomía por causa benigna durante los años 2006 a julio de 2012. Hubo un total de 379 histerectomías. De esta base de datos, se extrajeron las histerectomías vaginales y sus características de población, diagnósticos, complicaciones. Las variables se distribuyeron en porcentajes.

\section{RESULTADOS}

De las 379 histerectomías, el 17\% (n=64) se realizaron por vía abdominal y de estas últimas, en 31 pacientes se realizó la histerectomía abdominal por indicación de colposacropexia $(51,5 \%)$. La indicación de la vía abdominal en 33 pacientes (48,5\%) correspondió a histerectomía abdominal por contraindicación de vía vaginal. El 83\% $(n=315)$ se realizó por vía vaginal; sólo en 2 casos se abandonó la vía vaginal $(0,6 \%)$, para realizar el procedimiento por vía abdominal, debido a una lesión vascular y la otra a una lesión vesical imposible de corregir por vía vaginal.

Los principales diagnósticos pre quirúrgicos de histerectomía, tanto abdominal como vaginal, fueron: miomas (29\%), trastornos menstruales (24\%), prolapso de órganos pélvicos $(22 \%)$, adenomiosis sintomática $(7 \%)$ y otras causas $(19 \%)$.

El promedio de edad de las pacientes fue 57 años (rango: 32-88 años). El promedio de paridad fue 3,2 hijos (rango: 0-13 hijos), 16 pacientes eran nuligestas.
Hubo 6 casos de complicaciones intraoperatorias en las histerectomías vaginales (1,9\%): 4 cistostomías (1,2\%) (3 en pacientes sin cesárea y 1 en paciente con cesárea) y 2 lesiones vasculares $(0,6 \%)$.

De las 315 histerectomías vaginales, 90 (28,6\%) tenían antecedente de cesárea: 1 cesárea 73 casos $(81,1 \%), 2$ cesáreas 12 casos $(13,3 \%)$ y 3 cesáreas 5 casos $(5,5 \%)$. Se destaca que sólo una de las complicaciones descritas, ocurrió en una paciente con antecedente de tres cesáreas.

En relación con el seguimiento postoperatorio de las pacientes histerectomizadas por vía vaginal: se hospitalizaron 4 pacientes $(1,2 \%)$ con diagnóstico de absceso de cúpula vaginal, que requirieron drenaje vía vaginal y tratamiento antibiótico endovenoso, con recuperación total del cuadro al momento del alta hospitalaria.

\section{DISCUSIÓN}

Aunque la tasa de histerectomías totales ha permanecido estable, la distribución de la vía, es la que ha variado, pese a que han aumentado las histerectomías vaginales y las histerectomías laparoscópicas, la tasa de histerectomías abdominales, permanece en más del $66 \%$ de las pacientes (5), aun conociendo las ventajas de la cirugía vaginal.

Nuestros resultados difieren de los reportados en la literatura. Siendo conscientes de las ventajas de la cirugía vaginal, el $83 \%$ de las histerectomías independientemente del diagnóstico (causas benignas), se realizan por vía vaginal, a diferencia de la mayoría de otros centros en el mundo que reportan una incidencia de histerectomías vaginales sólo en el $22 \%$ (3). En nuestro medio existen reportes de histerectomía vaginal por causa benigna de un $34,1 \%$ y $36,9 \%(7,20)$.

En nuestro centro la vía vaginal es la elección, incluso en pacientes que tienen antecedentes de cesáreas, sin aumento de las complicaciones intraoperatorias. En nuestra experiencia el 28,6\% de las histerectomías vaginales tenían antecedente de cesárea y con una tasa de complicaciones similar a las reportadas en la literatura $(1,9 \%)(5,21)$ y menor del $7,9 \%$ reportado en nuestro medio $(7,21)$.

Las tasas de cistostomías accidentales comparados con reportes como los de Bhattacharya (16), son similares $(1,2 \%$ de nuestras pacientes vs $1,17 \%)$ y ligeramente menor a las reportadas por Ibeanu y cols (22), que reportan complicaciones vesicales del $2,2 \%$

En la revisión realizada, ninguna de las pacientes requirió unidad de terapia intensiva, no se presentaron lesiones intestinales, ni ocurrieron muertes. El porcentaje de lesiones vasculares en nuestra casuística $(0,6 \%)$, se asemeja al descrito en otras series que describen hemorragias en alrededor del $1,4 \%$ de los casos (23). Los trastornos 
sépticos se mencionan en el $2,7 \%$ y mortalidad hasta en el $0,18 \%$ de los casos (22).

En lo referente al abandono de la vía vaginal, este evento ocurrió sólo en dos pacientes $(0,6 \%)$ menor a lo reportado por trabajos recientes, que refieren una tasa de cambio de vía en el $2,6 \%$ de los casos (24).

Creemos necesaria la realización de trabajos prospectivos con el fin de aportar más datos acerca de este tema en nuestro medio. Creemos firmemente que por todos los beneficios conocidos, la vía vaginal debería ser la de elección en la gran mayoría de las pacientes sometidas a histerectomía. En este sentido, es labor de los centros docentes del país entrenar a los médicos en formación en la técnica de la histerectomía vaginal, para que así cada vez más pacientes se beneficien de una solución de bajo costo y morbilidad.

\section{CONCLUSIÓN}

Existe suficiente evidencia para concluir, que mientras sea posible, la vía vaginal, es la ruta ideal para la realización de la histerectomía, por menores complicaciones, rápida recuperación, curva de aprendizaje, costo efectividad y retorno de las pacientes a sus actividades rutinarias. Nuestros datos están en concordancia con las estadísticas nacionales e internacionales precedentes, en relación a las complicaciones intraoperatorias y la frecuencia de conversión de la vía vaginal a la abdominal, destacando en nuestra unidad el alto porcentaje de histerectomías practicadas por vía vaginal versus la abdominal, con baja incidencia de complicaciones intraoperatorias.

\section{REFERENCIAS}

1. Whiteman MK, Hillis SD, Jamieson DJ, Morrow B, Podgornik MN, Brett KM. Inpatient hysterectomy surveillance in the United States, 2000-2004. Am J Obstet Gynecol 2008;198:34.e1-7.

2. Garry R. Health economics of hysterectomy. Best Pract Res Clin Obstet Gynaecol 2005;19:451-65.

3. Wu JM, Wechter ME, Geller EJ, Nguyen TV, Visco AG. Hysterectomy rates in the United States, 2003. Obstet Gynecol 2007;110:1091-5

4. American College of Obstetricians and Gynecologists. ACOG committee opinion No. 444: Choosing the route of hysterectomy for benign disease. Obstet Gynecol 2009;114:1156-8.

5. Nieboer TE, Johnson N, Lethaby A, Tavender E, Curr E, Garry R, van Voorst S, Mol BW, Kluivers KB.. Surgical approach to hysterectomy for benign gynecological disease. Cochrane Database Syst Rev 2009;8(3). CD003677.pub 4.

6. Lucero M, Shah AD. Vaginal hysterectomy for the prolapsed uterus. Clin Obstet Gynecol 2010;53:26-39.

7. Salinas H, Pastén J, Naranjo B, Carmona S, Retamales B, Diaz G, Franulic L. Análisis clínico y económico de la histerectomía abdominal versus la histerectomía vaginal en el Hospital Clínico de la Universidad de Chile. Revisión de 2338 casos. Rev Chil Obstet Ginecol 2006;71:227-33.

8. Sheth SS. Vaginal hysterectomy as primary route for morbidly obese women. Acta Obstet Gynecol 2010;89:971-4.

9. Shaw A, Chelmow D. Vaginal hysterectomy. MEDSCAPE, August 2012.

10. Sheth SS. Prophylactic vaginal hysterectomy for benign hydatidiform mole. Int J GynecolObstet 2007;96:38-9.

11. Sheth SS, Paghdiwalla KP, Hajari AR. Vaginal route: a gynaecological route for much more than Agynaecological route for much more than hysterectomy. Best Pract Res ClinObstetGynaecol 2011;25:115-32.

12. Sharon A, Auslander R, Brandes-Klein O, Alter Z, Kaufman Y, Lissak A. Cystoscopy after total or subtotal laparoscopic hysterectomy: the value of a routine procedure. Gynecol Surg 2006;3:122-7.

13. Sheth SS. Adnexectomy for benign pathology at vaginal hysterectomy without laparoscopic assistance. BJOG 2002;109:1401-5.

14. Kulkarni MM, Rogers RG. Vaginal hysterectomy for benign disease without prolapse. Clin Obstet Gynecol 2010;53:5-16.

15. Dicker RC, Greenspan JR, Strauss LT, Cowart MR, Scally MJ, Peterson HB. Complications of abdominal and vaginal hysterectomy among women of reproductive age in the United States. The Collaborative Review of Sterilization. Am J ObstetGynecol 1982;144:841-8.

16. Bhattacharya M. Complications of vaginal hysterectomy (analysis of 1105 cases). J Postgrad Med 1978;24:221-5.

17. Muralidhar M, Glenn R. Vaginal hysterectomy for benign disease without prolapse clinical. Obstet Gynecol 2010;53:5-16

18. Kulkarni MM, Rogers RG. Vaginal hysterectomy for benign disease without prolapse. Clin Obstet Gynecol 2010;53:5-16.

19. Dicker RC, Greenspan JR, Strauss LT, Cowart MR, Scally MJ, Peterson HB. Complications of abdominal and vaginal hysterectomy among women of reproductive age in the United States. The Collaborative Review of Sterilization. Am J ObstetGynecol 1982;144:841-8.

20. Merino $P$, Doren A, Sáez N, Valenzuela $P$, Etchegaray A, Gejman R, Cuello M. Distribución de la patología quirúrgica del cuerpo uterino en mujeres chilenas sometidas a histerectomía en un hospital clínico universitario. Rev Chil Obstet Ginecol 2009;74:15-29.

21. Vesperinas G, Rondini C, Troncoso C, Morán B, Levancini $A$, Avilés $C$, Troncoso $F$. Histerectomia vaginal, abdominal y vaginal asistida por laparoscopia: un análisis prospectivo y aleatorio. Rev Chil Obstet Ginecol 2005;70:73-8.

22. Ibeanu OA, Chesson RR, Echols KT, Nieves M, Busangu $F$, Nolan TE. Urinary tract injury during hysterectomy based on universal cystoscopy. Obstet Gynecol 2009;113:6-10.

23. Muralidhar M, Glenn R Vaginal hysterectomy for benign disease without prolapsed. Clin Obstet Gynecol 2010;53:5-16.

24. Jones KA, Shepherd JP, Oliphant SS, Wang L, Bunker $\mathrm{CH}$, Lowder JL. Trends in inpatient prolapse procedures in the United States, 1979-2006. Am J Obstet Gynecol 2010;202:501.e1-7 